\title{
Perspectives on Platelet Heterogeneity and Host Immune Response in Coronavirus Disease 2019 (COVID-19)
}

Iván Parra-Izquierdo, $\mathrm{PhD}^{1,2}$ Joseph E. Aslan, $\mathrm{PhD}^{1,2,3}$

${ }^{1}$ Knight Cardiovascular Institute, Division of Cardiology, Department of Medicine, School of Medicine, Oregon Health and Science University, Portland, Oregon

2 Department of Biomedical Engineering, School of Medicine, Oregon Health and Science University, Portland, Oregon

${ }^{3}$ Department of Chemical Physiology and Biochemistry, School of Medicine, Oregon Health and Science University, Portland, Oregon

Semin Thromb Hemost 2020;46:826-830.
Address for correspondence Joseph E. Aslan, Knight Cardiovascular Institute, Division of Cardiology, Department of Medicine, School of Medicine, Oregon Health and Science University, 3303 SW Bond Avenue, Mail code $\mathrm{CH} 13 \mathrm{~B}$, Oregon Health \& Science University, Portland, OR 97239 (e-mail: aslanj@ohsu.edu).
Severe acute respiratory syndrome coronavirus 2 (SARS-CoV-2) infection and coronavirus disease 2019 (COVID-19) represent a global pandemic with largely uncharacterized but dire public health consequences. COVID-19 is now increasingly recognized as a thromboinflammatory disease, where thrombotic coagulopathy and intravascular coagulation are closely linked to mortality and clinical outcomes. ${ }^{1-4}$ As thrombocytopenia, systemic microvascular thrombosis, and elevated D-dimer levels reflect COVID-19 severity, ${ }^{1-4}$ cellular effectors of hemostasis and thrombosis-especially platelets-likely participate in COVID-19 pathogenesis. However, specific roles for platelets in COVID-19 as disease drivers, biomarkers, and therapeutic targets remain unspecified. Here, we highlight how platelets may be affected by COVID-19 in a manner supporting pathology, which offers insights into COVID-19 susceptibility, progression, and resolution. Like other viral infections and inflammatory states, COVID-19 likely involves alterations in platelet number, form, and function, or "platelet heterogeneity." 5,6 Knowledge gained over the past decade detailing mechanisms of platelet heterogeneity in inflammation and immune responses may help to gain ground in the battle against COVID-19. In turn, a surge of collaborative studies around COVID-19 pathogenesis may result in unique insights into platelet function critical to understanding and managing other inflammatory disease states.

\section{Platelet Heterogeneity in Infection, Host Response, and COVID-19}

Platelets are well known as specialized, peripheral blood cells essential to hemostasis and vascular health, but they also causally contribute to acute and chronic pathologies, including thrombosis and vascular inflammation. ${ }^{7}$ Translational efforts over the past decade, aiming to determine disease-specific platelet activities, have identified heterogeneous platelet phenotypes in the laboratory as well as in the clinic associated with inflammatory, thrombotic, and other conditions, ${ }^{5,8-10}$ including pathologies driven by a variety of viral infections. ${ }^{11-13}$ The mechanistic basis for how "proinflammatory," "procoagulant," and other potentially pathogenic platelet phenotypes come about remains vague. Consequently, efforts to develop effective therapeutic, diagnostic, and preventive strategies against maladaptive platelet mechanisms in thromboinflammatory pathologies remain delayed. As such, mechanistic knowledge gaps around platelet function in COVID-19 represent an area of unmet clinical need, but also provide opportunities for studying platelet heterogeneity in a critically relevant inflammatory context.

Heterogeneity in platelet phenotype as well as number (i.e., thrombocytopenia) are general hallmarks of viral infections, where a complex interplay between viral replication cycles, immune evasion, host response, and other mechanisms affect platelet function and turnover. ${ }^{14}$ Over the course of viral infection, interactions between platelet receptors and specific pathogen-associated molecular patterns (PAMPs) can promote platelet heterogeneity through innate immunity receptors, including toll-like receptors (TLRs), which sense PAMPs at the platelet plasma membrane or become activated in platelet endosomes upon PAMP internalization. ${ }^{15}$ For example, human cytomegalovirus directly binds to and activates platelets through TLR2 to promote inflammation and tissue injury. ${ }^{16}$ Likewise, following published online September 3, 2020
Issue Theme Maintaining Hemostasis and Preventing Thrombosis in COVID-19 -Part I; Guest Editors: Emmanuel J. Favaloro, PhD, FFSc (RCPA), and Giuseppi Lippi, MD.
Copyright $\odot 2020$ by Thieme Medical Publishers, Inc., 333 Seventh Avenue, New York, NY 10001, USA. Tel: +1(212) 760-0888.
DOI https://doi.org/ 10.1055/s-0040-1715093. ISSN 0094-6176. 
secretion into the bloodstream, the dengue virus nonstructural protein (NS1) triggers thromboinflammatory responses in a platelet TLR4-dependent manner. ${ }^{17}$ Currently, there is no evidence of SARS-CoV-2 sequestration or replication in megakaryocytes or platelets nor a widespread presence of SARS-CoV-2 nucleic acids or gene products in the circulation. However, SARS-CoV-2 N1 mRNA has been detected in platelets from a small number of COVID-19 patients (2/25 tested), despite no expression of the SARSCoV-2 receptor ACE2 by platelets. ${ }^{18}$ Nonetheless, platelets are fully equipped to interact with and likely to internalize SARS-CoV-2 particles or components that may enter circulation in a manner relevant to the initial stages of infection, endothelial cell (EC) disruption, and COVID-19 pathogenesis, as recently detailed by Koupenova. ${ }^{19}$

Severe SARS-CoV-2 infection is now characterized by a defective type I interferon (IFN) response, as well as an especially exaggerated production and secretion of interleukin (IL)-6 together with IL-1, IL-8, IFN- $\gamma$, tumor necrosis factor- $\alpha$ (TNF- $\alpha$ ), and other immune modulators. ${ }^{20}$ This COVID-19 "cytokine storm" is suspected of obfuscating platelet function in a manner further promoting platelet heterogeneity, inflammation, and vascular dysfunction. As a key component of interest to COVID-19 pathology, IL-6 is already known to promote platelet activation in inflammatory contexts, but mechanisms underlying IL-6 platelet priming still remain unclear. For instance, while platelets express the IL-6 receptor subunit IL-6R $\beta$ (gp130), they do not express IL-6R $\alpha$ and require exogenous addition of soluble sIL-6R $\alpha$ to activate Jak/STAT and MAPK signaling ex vivo. ${ }^{21}$ In vivo, however, platelets may respond to IL-6 through interactions with leukocytes that bring IL-6R $\alpha$ in close proximity to platelets in a manner related to models of platelet-leukocyte interactions in COVID-19 proposed by Merad and Martin, $^{22}$ and that may be targeted by agents such as tocilizumab. ${ }^{23}$ Such mechanisms are supported by recent studies demonstrating upregulated MAPK signaling, and increased platelet reactivity and platelet-leukocyte aggregation in COVID-19 patients. ${ }^{18}$ An increased cross-talk between platelets and leukocytes has also been linked to other features of hypercoagulability in COVID-19 pathology, where platelet-monocyte aggregates fuel tissue factor (TF) expression, and platelet-neutrophil interactions contribute to microthrombosis and acute respiratory distress syndrome (ARDS). ${ }^{24,25}$

Following cytokine storm and exacerbated inflammation, injured tissues may release damage-associated molecular patterns (DAMPs) into circulation, ${ }^{20}$ typically including high-mobility group box (HMGB1), S100 family, and histone proteins. DAMPs may be detected by innate immune receptors on platelets, including TLRs, to bring about platelet heterogeneity supporting inflammation, coagulation, and sepsis (-Fig. 1). In addition to TLR responses, proteomics and clinical studies demonstrate robust complement system activation in severe platelet-related COVID-19 pathologies. $^{26-29}$ Activation of complement receptor on platelets may prime specific platelet phenotypes and promote platelet-leukocyte interactions associated with microvascular thrombosis and platelet consumption in COVID-19. ${ }^{30,31}$ Antibody-mediated immune thrombocytopenia (ITP) mechanisms can also fuel platelet heterogeneity through platelet Fc receptor activation and platelet destruction in COVID-19. 1,32 Whether or not platelet heterogeneity and thrombocytopenia in severe COVID-19 follow from these innate and adaptive immune responses or an inhibition of platelet production remains to be clarified. ${ }^{31}$ Moreover, it remains to be determined whether restoring platelet counts in severe COVID-19 patients may help or hinder the host resolution of SARS-CoV-2 infection. ${ }^{31}$

\section{Platelet Activation and Thrombosis in COVID-19}

Beyond interactions with circulating immune modulators and endogenous damage signals, factors from inflamed ECs also likely to encourage platelets to take on inflammatory phenotypes biased toward thrombosis in contexts such as COVID-19, especially if platelets and other immune cells are already primed by chronic disease. ${ }^{34,35}$ For instance, von Willebrand factor (VWF) expression and multimerization on ECs are generally associated with vascular inflammation, ${ }^{36}$ and VWF has been reported to be increased in COVID-19 in parallel with hypercoagulability and disease severity. 37,38 Endothelial damage and vascular leakage also generally support platelet adhesion and activation through classical hemostatic responses to extracellular matrix molecules, where the platelet receptor GPVI rapidly activates platelet activation programs in response to collagen (- Fig. 1) ${ }^{39}$ In addition to hemostatic interactions, platelets also interact directly with the endothelium and modulate the adhesion and infiltration of immune cells. For example, platelets play a fundamental role in regulating endothelial inflammation and permeability by synthesizing and secreting IL-1 to progress inflammatory diseases. ${ }^{40}$ Interestingly, proteomics studies have found upregulated, downregulated, and unaltered levels of several proteins known to be released by platelets in plasma and serum of COVID-19 patients in a manner reflective of disease severity. ${ }^{26,27}$ This seemingly differential platelet releasate in COVID-19 may be due to lower platelet counts or indicative of "exhausted" platelet phenotypes similarly found in other viral infection contexts. ${ }^{5}$ However, more specific mechanistic studies are needed to better determine whether and how platelet secretion may be altered in COVID-19.

The likely roles of platelet priming, heterogeneity, and (hyper)activation in COVID-19 suggest that antiplatelet agents may have protective effects or improve clinical outcomes in COVID-19. These possibilities have been extensively considered by several investigators, including Bikdeli et al and the Global COVID-19 Thrombosis Collaborative Group (-Fig. 1). ${ }^{41}$ In addition to targeting inflammation or immune responses, several trials are now underway to determine whether and how aspirin, P2Y12 inhibitors (i.e., clopidogrel, ticagrelor), and other platelet-targeted agents may benefit COVID-19 patients. Some early, small-scale studies suggest that agents such as dipyridamole may promote the clinical 


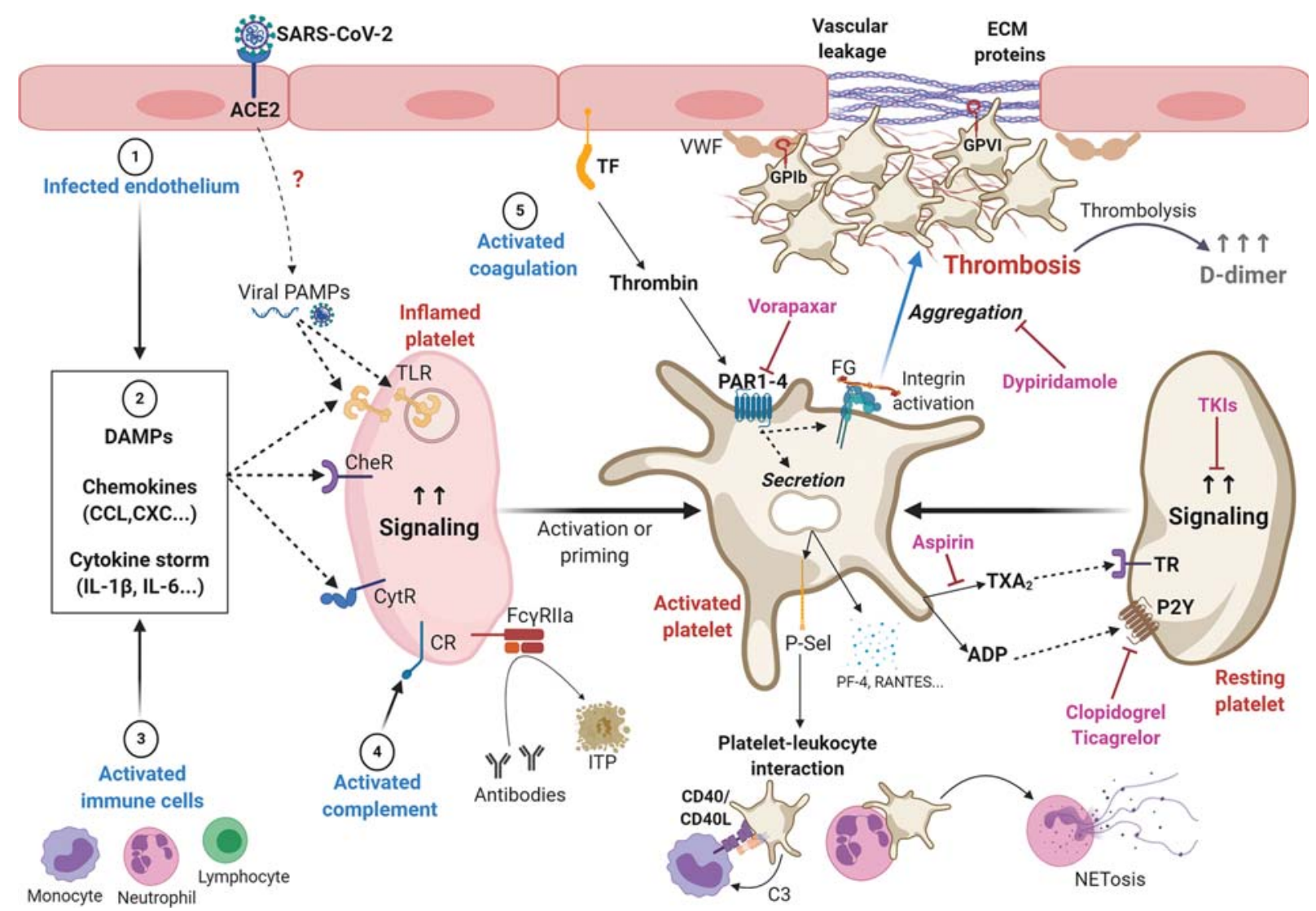

Fig. 1 Potential mechanisms contributing to platelet heterogeneity, inflammation, and thrombosis in coronavirus disease 2019 (COVID-19). Following SARS-CoV-2 infection through ACE2 receptors, endothelial cells may upregulate a variety of host responses (1), including secretion of ILs, chemokines, and DAMPs (2). Viral replication products may also be secreted into the circulation and act as PAMPs. Activated immune cells are also a source of ILs, chemokines, and DAMPs (3). These ligands may activate a variety of receptors on platelets, including TLR, CytR, and CheR. Immunity-associated pathways associated with the complement system are also evident in COVID-19, which may support platelet activation through CR on platelets (4). Other immune responses following FCYRIla antibody ligation may also support platelet activation or platelet destruction and ITP. In this inflammatory context of COVID-19, platelets may more readily adhere to VWF on dysfunctional or damaged endothelium, which is likely upregulated in COVID-19 at sites of vascular leakage. After adhering to endothelial VWF through GPIb, platelets are readily activated by ECM proteins such as collagen, which can drive immunothrombosis through the immunotyrosine (ITAM) receptor GPVI. ADP secretion and thromboxane A2 (TxA2) synthesis amplify signaling events supporting platelet activation through TR and purinergic receptors (P2Y). Thrombin generation through TF pathways also supports the activation of PARs to establish procoagulant platelets that further progress thrombosis and fibrin formation (5). Immunothrombosis in COVID-19 may be further progressed by inflammatory factors secreted from platelets-including P-selectin, platelet factor 4 (PF4, CXCL4) RANTES (CCL5), IL-18, CD40L (CD154) and VEGF-to support platelet-leukocyte interactions as well as neutrophil extracellular traps (NETosis). Altogether, host immune responses and classic platelet hemostatic programs may come together and further fuel vascular inflammation, thrombosis, and mortality in COVID-19 through dysregulated platelet adhesion, secretion, integrin activation, and aggregation. Accordingly, platelet-directed drugs (i.e., aspirin, clopidogrel, dipyridamole, TKIs) may offer therapeutic options to dampen thrombus formation and improve outcomes in COVID-19. ACE2, angiotensin-converting enzyme 2; ADP, adenosine diphosphate; CheR, chemokine receptors; CR, complement receptor; CytR, cytokine receptors; DAMP, damage associated molecular pattern; ECM, extracellular matrix; FCYRIla, Fc gamma receptor Ila; GP, glycoprotein; IL, interleukin; ITP, immune thrombocytopenia; PAMP, pathogen-associated molecular pattern; PAR, protease activated receptor; SARS-CoV-2, severe acute respiratory syndrome coronavirus 2; TF, tissue factor; TKI, tyrosine kinase inhibitor; TR, thromboxane receptor; TLR, toll-like receptor; VWF, von Willebrand factor.

recovery of severe COVID-19 ${ }^{42}$; however, other studies find no benefit for pretreatment with antiplatelet agents in severe cases of COVID-19, ${ }^{43}$ and large-scale extended trials are required to better understand the role of antiplatelet agents in COVID-19 as well as in vascular inflammation. ${ }^{44}$ Many other agents that may specifically interfere with thromboinflammatory platelet responses are also of interest, including tyrosine kinase inhibitors that block platelet GPVI signaling (i.e., ibrutinib, acalabrutinib). ${ }^{39,45,46}$ Intrinsic and extrinsic coagulation pathways that regulate thrombin formation and promote procoagulant platelet phenotypes are also targets of interest in COVID-19. ${ }^{41,47}$

\section{Platelet Phenotype and Function in COVID- 19 and Beyond}

Interestingly, several COVID-19 comorbidities are already associated with platelet heterogeneity as well as poor outcomes in COVID-19, including metabolic syndrome, diabetes ${ }^{48}$ gut dysbiosis, ${ }^{49}$ cardiovascular disease, ${ }^{50}$ cancer, and aging. ${ }^{5,6}$ Whether these conditions represent primed platelet states that may aggravate COVID-19 illness will become apparent as platelet activation mechanisms in inflammation and SARS-CoV-2 infection are further worked out. It is now recognized that alterations in platelet form and 
function can occur in a manner likely supporting specific disease states ${ }^{5,6}$; however, it remains unclear how these changes come about in platelets at a molecular level in inflammatory diseases in general, and in specific cases such as COVID-19. To this end, quantitative phosphoproteomics profiling and pathway mapping may offer a means to define platelet phenotypes in specific contexts, ${ }^{51}$ and collaborative efforts such as the COVID-19 Disease Map Initiative may help to specify molecular features of platelet phenotypes in COVID-19. ${ }^{52}$ Regardless of current and future prospects of defining biomarkers and platelet phenotypes specific to COVID-19, several lines of evidence point to platelets as important regulators of dysregulated inflammation and coagulation in COVID-19. Ongoing and future studies of platelets in inflammation and COVID-19 will better determine whether and how platelet function is beneficial and detrimental to, as well as informative of, disease progression and outcomes in SARS-CoV-2 infection.

\section{Funding}

This work is supported by the National Institutes of Health (grant R01HL146549).

\section{Conflict of Interest}

The authors report no conflicts of interest.

\section{References}

1 Larsen JB, Pasalic L, Hvas AM. Platelets in coronavirus disease 2019. Semin Thromb Hemost 2020 (e-pub ahead of print) . Doi: 10.1055/s-0040-1710006

2 Al-Samkari H, Karp Leaf RS, Dzik WH, et al. COVID and coagulation: bleeding and thrombotic manifestations of SARS-CoV2 infection. Blood 2020 (e-pub ahead of print) . Doi: 10.1182/ blood.2020006520

3 Fox SE, Akmatbekov A, Harbert JL, Li G, Quincy Brown J, Vander Heide RS. Pulmonary and cardiac pathology in African American patients with COVID-19: an autopsy series from New Orleans. Lancet Respir Med 2020 (e-pub ahead of print) . Doi: 10.1016/ S2213-2600(20)30243-5

4 Chen G, Wu D, Guo W, et al. Clinical and immunological features of severe and moderate coronavirus disease 2019. J Clin Invest 2020;130(05):2620-2629

5 Baaten CCFMJ, Ten Cate H, van der Meijden PEJ, Heemskerk JWM. Platelet populations and priming in hematological diseases. Blood Rev 2017;31(06):389-399

6 van der Meijden PEJ, Heemskerk JWM. Platelet biology and functions: new concepts and clinical perspectives. Nat Rev Cardiol 2019;16(03):166-179

7 Aslan JE. Platelet shape change. In: Gresele P, López J, Kleiman N, Page C eds, Platelets in Thrombotic and Non-Thrombotic Disorders. Cham, Switzerland: Springer; 2017

8 Berger JS, Becker RC, Kuhn C, Helms MJ, Ortel TL, Williams R. Hyperreactive platelet phenotypes: relationship to altered serotonin transporter number, transport kinetics and intrinsic response to adrenergic co-stimulation. Thromb Haemost 2013;109 (01):85-92

9 Agbani EO, Poole AW. Procoagulant platelets: generation, function, and therapeutic targeting in thrombosis. Blood 2017;130 (20):2171-2179

10 Munnix IC, Cosemans JM, Auger JM, Heemskerk JW. Platelet response heterogeneity in thrombus formation. Thromb Haemost 2009;102(06):1149-1156
11 Koupenova M, Vitseva O, MacKay CR, et al. Platelet-TLR7 mediates host survival and platelet count during viral infection in the absence of platelet-dependent thrombosis. Blood 2014;124(05): 791-802

12 Koupenova M, Clancy L, Corkrey HA, Freedman JE. Circulating platelets as mediators of immunity, inflammation, and thrombosis. Circ Res 2018;122(02):337-351

13 Katz JN, Kolappa KP, Becker RC. Beyond thrombosis: the versatile platelet in critical illness. Chest 2011;139(03):658-668

14 Page MJ, Pretorius E. A champion of host defense: a generic largescale cause for platelet dysfunction and depletion in infection. Semin Thromb Hemost 2020;46(03):302-319

15 D' Atri LP, Schattner M. Platelet toll-like receptors in thromboinflammation. Front Biosci 2017;22:1867-1883

16 Assinger A, Kral JB, Yaiw KC, et al. Human cytomegalovirusplatelet interaction triggers toll-like receptor 2-dependent proinflammatory and proangiogenic responses. Arterioscler Thromb Vasc Biol 2014;34(04):801-809

17 Quirino-Teixeira AC, Rozini SV, Barbosa-Lima G, et al. Inflammatory signaling in dengue-infected platelets requires translation and secretion of nonstructural protein 1. Blood Adv 2020;4(09): 2018-2031

18 Manne BK, Denorme F, Middleton EA, et al. Platelet gene expression and function in COVID-19 patients. Blood 2020. Doi: 10.1182/blood.2020007214 [epub ahead of print]

19 Koupenova M. Potential role of platelets in COVID-19: implications for thrombosis. research and practice in thrombosis and haemostasis. Res Pract Thromb Haemost 2020;4(05): 737-740

20 Tang D, Comish P, Kang R. The hallmarks of COVID-19 disease. PLoS Pathog 2020;16(05):e1008536

21 Houck KL, Yuan H, Tian Y, et al. Physical proximity and functional cooperation of glycoprotein 130 and glycoprotein VI in platelet membrane lipid rafts. J Thromb Haemost 2019;17(09): 1500-1510

22 Merad M, Martin JC. Pathological inflammation in patients with COVID-19: a key role for monocytes and macrophages. Nat Rev Immunol 2020;20(06):355-362

23 Xu X, Han M, Li T, et al. Effective treatment of severe COVID-19 patients with tocilizumab. Proc Natl Acad Sci U S A 2020;117(20): 10970-10975

24 Hottz ED, Azevedo-Quintanilha IG, Palhinha L, et al. Platelet activation and platelet-monocyte aggregates formation trigger tissue factor expression in severe COVID-19 patients. Blood 2020. Doi: 10.1182/blood.2020007252 [epub ahead of print]

25 Middleton EA, He XY, Denorme F, et al. neutrophil extracellular traps (NETs) contribute to immunothrombosis in COVID-19 acute respiratory distress syndrome. Blood 2020. (epub ahead of print). Doi: 10.1182/blood.2020007008

26 Shen B, Yi X, Sun Y, et al. Proteomic and metabolomic characterization of COVID-19 Patient Sera. Cell 2020 (e-pub ahead of print). Doi: 10.1016/j.cell.2020.05.032

27 Messner CB, Demichev V, Wendisch D, et al. Ultra-high-throughput clinical proteomics reveals classifiers of COVID-19 infection. Cell Syst 2020. (e-pub ahead of print). Doi: 10.1016/j. cels.2020.05.012

28 Magro C, Mulvey JJ, Berlin D, et al. Complement associated microvascular injury and thrombosis in the pathogenesis of severe COVID-19 infection: a report of five cases. Transl Res 2020;220:1-13

29 Noris M, Benigni A, Remuzzi G. The case of complement activation in COVID-19 multiorgan impact. Kidney Int 2020 (e-pub ahead of print) . Doi: 10.1016/j.kint.2020.05.013

30 Eriksson O, Mohlin C, Nilsson B, Ekdahl KN. The human platelet as an innate immune cell: interactions between activated platelets and the complement system. Front Immunol 2019; 10:1590 
31 Qiu J, Ma J, Zhang S, Han J, Liu S. Promoting platelets is a therapeutic option to combat severe viral infection of the lung. Blood Adv 2020;4(08):1640-1642

32 Bomhof G, Mutsaers PGNJ, Leebeek FWG, et al. COVID-19-associated immune thrombocytopenia. Br J Haematol 2020 (e-pub ahead of print). Doi: 10.1111/bjh.16850

33 Xu P, Zhou Q, Xu J. Mechanism of thrombocytopenia in COVID-19 patients. Ann Hematol 2020;99(06):1205-1208

34 Matacic C. Blood vessel injury may spur disease's fatal second phase. Science 2020;368(6495):1039-1040

35 Teuwen LA, Geldhof V, Pasut A, Carmeliet P. COVID-19: the vasculature unleashed. Nat Rev Immunol 2020 (e-pub ahead of print) . Doi: 10.1038/s41577-020-0343-0

36 Chen J, Chung DW. Inflammation, von Willebrand factor, and ADAMTS13. Blood 2018;132(02):141-147

37 Panigada M, Bottino N, Tagliabue P, et al. Hypercoagulability of COVID-19 patients in intensive care unit. A report of thromboelastography findings and other parameters of hemostasis. J Thromb Haemost 2020 (e-pub ahead of print) . Doi: 10.1111/ jth. 14850

38 Helms J, Tacquard C, Severac F, et al; CRICS TRIGGERSEP Group (Clinical Research in Intensive Care and Sepsis Trial Group for Global Evaluation and Research in Sepsis). High risk of thrombosis in patients with severe SARS-CoV-2 infection: a multicenter prospective cohort study. Intensive Care Med 2020;46:1089-1098

39 Rayes J, Watson SP, Nieswandt B. Functional significance of the platelet immune receptors GPVI and CLEC-2. J Clin Invest 2019; 129(01):12-23

40 Gawaz M, Langer H, May AE. Platelets in inflammation and atherogenesis. J Clin Invest 2005;115(12):3378-3384

41 Bikdeli B, Madhavan MV, Gupta A, et al; Global COVID-19 Thrombosis Collaborative Group. Pharmacological agents targeting thromboinflammation in COVID-19: review and implications for future research. Thromb Haemost 2020 (e-pub ahead of print) . Doi: $10.1055 / \mathrm{s}-0040-1713152$
42 Liu X, Li Z, Liu S, et al. Potential therapeutic effects of dipyridamole in the severely ill patients with COVID-19. Acta Pharm Sin B 2020 (e-pub ahead of print) . Doi: 10.1016/j.apsb.2020.04.008

43 Russo V, Di Maio M, Attena E, et al. Clinical impact of preadmission antithrombotic therapy in hospitalized patients with COVID-19: a multicenter observational study. Pharmacol Res 2020;159:104965

44 Libby P. Interleukin- 1 beta as a target for atherosclerosis therapy: biological basis of CANTOS and beyond. J Am Coll Cardiol 2017;70 (18):2278-2289

45 Roschewski M, Lionakis MS, Sharman JP, et al. Inhibition of Bruton tyrosine kinase in patients with severe COVID-19. Sci Immunol 2020;5(48):eabd0110

46 Treon SP, Castillo JJ, Skarbnik AP, et al. The BTK inhibitor ibrutinib may protect against pulmonary injury in COVID-19-infected patients. Blood 2020;135(21):1912-1915

47 Shatzel JJ, DeLoughery EP, Lorentz CU, et al. The contact activation system as a potential therapeutic target in patients with COVID19. Res Pract Thromb Haemost 2020;4(04):500-505

48 Zhu L, She ZG, Cheng X, et al. Association of blood glucose control and outcomes in patients with COVID-19 and pre-existing type 2 diabetes. Cell Metab 2020;31(06):1068-1077

49 Parra-Izquierdo I, Bradley R, Aslan JE. Platelets get gutted by PAG. Platelets 2020;1-3:1-3

50 Gupta AK, Jneid H, Addison D, et al. Current perspectives on Coronavirus 2019 (COVID-19) and cardiovascular disease: A white paper by the JAHA editors. J Am Heart Assoc 2020;9(12): e017013

51 Babur Ö, Melrose AR, Cunliffe JM, et al. Phosphoproteomic quantitation and causal analysis reveal pathways in GPVI/ITAM-mediated platelet activation programs. Blood 2020. Doi: 10.1182/ blood.2020005496 [epub ahead of print]

52 Ostaszewski M, Mazein A, Gillespie ME, et al. COVID-19 Disease Map, building a computational repository of SARS-CoV-2 virushost interaction mechanisms. Sci Data 2020;7(01):136 